\title{
Volumetric Source Model to Predict Productivity of Fractured Horizontal Well in Naturally Fractured Reservoirs
}

\author{
Tianhui Wang ${ }^{1}$, Haitao $\mathrm{Li}^{2}$, Junchao Wang ${ }^{2,3, *}$, Yunlin $\mathrm{Jia}^{1}$ and Lifeng Liu ${ }^{1}$ \\ ${ }^{I}$ CNOOC China Limited QHD32-6 Operating Company, Tianjin, 300000, P. R. China, ${ }^{2}$ State Key Laboratory of Oil and \\ Gas Reservoir Geology and Exploitation, Southwest Petroleum University, Chengdu, 610500, P. R. China; ${ }^{3}$ Engineering \\ Technology Research Institute of Xinjiang Oilfield Company, Karamay, 834000, P. R. China
}

\begin{abstract}
To predict productivity of fractured horizontal wells with different completion methods, the flow models of reservoir and hydraulic fractures are developed by utilizing volumetric source idealization, while the models solved by taking orthogonal transformation. Compared to traditional point source functions, volumetric source function has many advantages in properties of function and programming calculation. A practical example of fractured horizontal well of open-hole and cased-hole is simulated, result shows flow rates of two cases have significant difference at early time, since benefit of production of horizontal well; but it also causes additional pressure, which has disadvantage in the productivity. The difference of productivity of fractured horizontal wells with different completion is decided by the interaction of horizontal well's production and additional pressure drop, which has advantage and disadvantage in the open-hole case respectively. As a result, methods to cause additional pressure drop more seriously such as increasing number, length or conductivity of hydraulic fractures, would reduce the difference; methods to enhance horizontal well's production such as better reservoir flow capacity, would raise the difference.
\end{abstract}

Keywords: Cased-hole horizontal well, multiple-fractured horizontal well, naturally fractured reservoir, open-hole horizontal well, productivity, volumetric source model.

\section{INTRODUCTION}

Hydraulic fracturing is widely employed to improve productivity of horizontal wells. Methods to predict the productivity of fractured horizontal wells become invaluable tools to optimize the hydraulic fracturing and determine productivity of horizontal wells. While main analytical [1-4] and semi-analytical models [5-8] are ignoring the direct flow to horizontal well, which fitted to hydraulic fractured casedhole horizontal wells. But, for hydraulic fractured open-hole horizontal wells, ignoring the flow to horizontal well will cause tremendous error, especially at early time [9-13].

Since Gringarten and Ramey [14] introduced to solve transient flow problems for the first time, various source functions [5-13] are developed to simulate pressure behavior and productivity of fractured horizontal wells. But most works have been focusing on point source functions which neglected the flow in source and its volume. While Ogunsanya et al. [15, 16] pointed out that source functions have computational problems and the no volume assumption may not be adequate in some specific situations, then revisited the concept of volumetric source and presented a new "solid bar source" model. Amini et al. [17-20] developed distributed volumetric source (DVS) model of homogeneous reservoirs, and validate the applications in vertical well, horizontal well and fractured vertical fractured well with well-known existing solutions. He also demonstrated the application of DVS

\footnotetext{
*Address correspondence to this author at the Engineering Technology Research Institute of Xinjiang Oilfield Company, Karamay, 834000 P. R. China; Tel: +8615739716313; Fax: 0990-6222694;

E-mail: wangjunchao2012@126.com
}

model in fractured horizontal wells and emphasized advantages of the method of volumetric source. The solution of volumetric source model does not suffer the problem of inherent singularity; pressure can be calculated at any point. Meanwhile, it is more adjustable than point source solutions; the solution is always the same formation, no matter the types of the source.

This study is focused on the derivation and application of volumetric source models in naturally fractured reservoir and hydraulic fractures for open-hole horizontal wells and casedhole horizontal wells. Unsteady volumetric source model is generalized for fractured reservoir. Then a new steady volumetric source model is employed to calculate pressure drop in hydraulic fractures for finite-conductivity assumption. Both of the two models can be solved through orthogonal transformation. Productivity of fractured horizontal wells for open-hole or cased-hole is calculated by coupling the two models. An example of fractured horizontal well is studied, difference between open-hole case and cased-hole case is illustrated, and factors of hydraulic fractures and reservoir, affecting the productivity are simulated.

\section{VOLUMETRIC SOURCE MODEL}

\subsection{Assumption}

The porous media is assumed to be dual-porosity reservoir which shaped as a box with closed boundaries, and size of the reservoir is $\left(x_{e}, y_{e}, z_{e}\right)$. A volumetric source produces oil in the reservoir with constant rate $q$, the central coordinate of the source is $\left(c_{x}, c_{y}, c_{z}\right)$, and size is $\left(2 w_{x}, 2 w_{y}, 2 w_{z}\right)$. Fig. (1) shows the schematic of the system. 


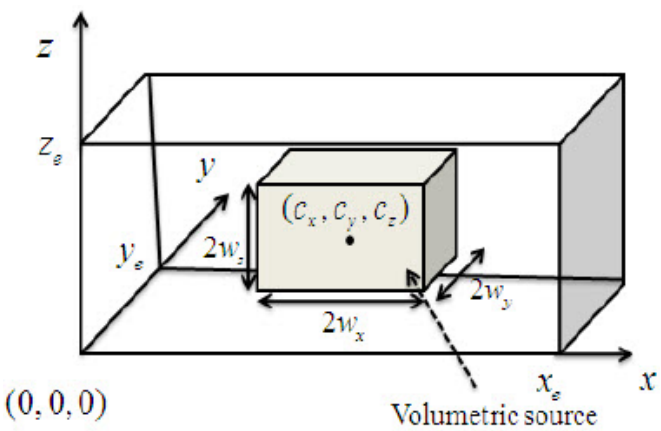

Fig. (1). Schematic of volumetric source system.

The rock and oil are considered to be slightly compressible with constant compressibility, and flow is assumed to be isothermal and follow Darcy's low.

\subsection{Mathematical Model}

In dual-porosity idealizations, Warren and Root introduced the dual-porosity model in term of bulk properties. If $C$, denotes an intrinsic property of medium $!=m$ (matrix) or $f$ (natural fractures), then bulk property of medium $\varsigma$ is,

$C_{\varsigma B}=C_{\varsigma} V_{\varsigma}$

where $V_{\varsigma}$ is the ratio of volume of medium $\varsigma, V_{m}+V_{f}=1$.

Characteristics of the matrix and natural fracture system are incorporated by the storativity $\omega$ and the flow capacity ratio $\lambda$ is defined as,

$\omega=\frac{\left(\phi c_{t}\right)_{f B}}{\left(\phi c_{t}\right)_{m B}+\left(\phi c_{t}\right)_{f B}}, \quad \lambda=\alpha \frac{k_{m B}}{k_{f B}} L^{2}$

where $L$ is 1 an arbitrary reference length(m), in this paper $L=\left(x_{e} y_{e} z_{e}\right)^{\overline{3}} ; \alpha$ is the inter-porosity shape factor $\left(1 / \mathrm{m}^{2}\right)$; $k_{\varsigma}$ and $\left(\phi c_{t}\right)_{\varsigma}$ are permeability $\left(\mu \mathrm{m}^{2}\right)$ and storage $\left(\mathrm{MPa}^{-1}\right)$ of medium $\varsigma$.

Dimensionless variables are defined as,

$P_{D}=\frac{86.4 k_{f B} L\left(p_{i}-p\right)}{B q_{c} \mu}, q_{D}=\frac{q}{q_{c}}, l_{D}=\frac{l}{L}$,

$t_{D}=\frac{3.6 k_{f B} t}{\mu L^{2}\left[\left(\phi c_{t}\right)_{m B}+\left(\phi c_{t}\right)_{f B}\right]}$

where $q_{c}$ is an arbitrary reference production $\operatorname{rate}\left(\mathrm{m}^{3} / \mathrm{d}\right) ; l$ represents coordinate of direction $x, y, z ; \mu$ is viscosity( $\mathrm{mPa} \cdot \mathrm{s}) ; p_{i}$ is initial pressure $(\mathrm{MPa}) ; B$ is oil formation factor.

Volumetric source model is,

$(1-\omega) \frac{\partial P_{m D}}{\partial t_{D}}+\lambda\left(P_{m D}-P_{f D}\right)=0$

$\frac{\partial^{2} P_{f D}}{\partial x_{D}^{2}}+\frac{\partial^{2} P_{f D}}{\partial y_{D}^{2}}+\frac{\partial^{2} P_{f D}}{\partial z_{D}^{2}}+\lambda\left(P_{m D}-P_{f D}\right)-\frac{q_{D} h\left(x_{D}, y_{D}, z_{D}\right)}{V_{\text {source }, D}}=\omega \frac{\partial P_{f D}}{\partial t_{D}}$

$\left.P_{m D}\right|_{t_{D}=0}=\left.P_{f D}\right|_{t_{D}=0}=0$ $\left.\frac{\partial P_{f D}}{\partial l_{D}}\right|_{l_{D}=0}=0$
$\left.\frac{\partial P_{f D}}{\partial l_{D}}\right|_{l_{D}=l_{e D}}=0$

where,

$V_{\text {source }, D}=8 w_{x D} w_{y D} w_{z D}$

$h\left(x_{D}, y_{D}, z_{D}\right)=\left[H\left(x_{D}-c_{x D}-w_{x D}\right)-\right.$

$\left.H\left(x_{D}-c_{x D}+w_{x D}\right)\right] \times\left[H\left(y_{D}-c_{y D}-w_{y D}\right)\right.$

$\left.-H\left(y_{D}-c_{y D}+w_{y D}\right)\right]$

$\times\left[H\left(z_{D}-c_{z D}-w_{z D}\right)-H\left(z_{D}-c_{z D}+w_{z D}\right)\right]$

$H\left(x-x_{0}\right)$ is Heaviside function:

$H\left(x-x_{0}\right)=\left\{\begin{array}{ll}1 & x>x_{0} \\ 0 & x \leq x_{0}\end{array}\right.$ 。

\subsection{Orthogonal Transformation}

According to initial-boundary value problem (1)-(5), the boundary value problem of characteristic function is,

$\frac{\partial^{2} E}{\partial x_{D}^{2}}+\frac{\partial^{2} E}{\partial y_{D}^{2}}+\frac{\partial^{2} E}{\partial z_{D}^{2}}+\gamma E=0$

$\left.\frac{\partial E}{\partial l_{D}}\right|_{l_{D}=0}=0$

$\left.\frac{\partial E}{\partial l_{D}}\right|_{l_{D}=l_{e D}}=0$ (8) is,

Characteristic function of boundary value problem (6)-

$$
E_{k, m, n}\left(x_{D}, y_{D}, z_{D}\right)=\cos \frac{k \pi}{x_{e D}} x_{D} \cos \frac{m \pi}{y_{e D}} y_{D} \cos \frac{n \pi}{z_{e D}} z_{D}
$$

Characteristic value is,

$\gamma=\left(\frac{k \pi}{x_{e D}}\right)^{2}+\left(\frac{m \pi}{y_{e D}}\right)^{2}+\left(\frac{n \pi}{z_{e D}}\right)^{2}$

Norm of characteristic function is,

$\left\|E_{k, m, n}\left(x_{D}, y_{D}, z_{D}\right)\right\|^{2}=\frac{2^{\delta_{k k}+\delta_{m m}+\delta_{n n}}}{8} x_{e D} y_{e D} z_{e D}$

$\delta_{k k}= \begin{cases}1 & k=0 \\ 0 & k \neq 0\end{cases}$

The orthogonal transformation is defined for such a complete orthogonal function system, 
$\tilde{P}_{f}=T\left(P_{f D}\right)=\int_{\Omega_{D}} E_{k, m, n}\left(x_{D}, y_{D}, z_{D}\right) P_{f D} d V_{D}$

Where inverse transformation formula is,

$P_{f D}=\sum_{k=0}^{\infty} \sum_{m=0}^{\infty} \sum_{n=0}^{\infty} \frac{E_{k, m, n}}{\left\|E_{k, m, n}\right\|^{2}} \tilde{P}_{f}$

After orthogonal transformation, the initial-boundary value (1)-(5) can be described as following initial value problem,

$\left\{\begin{array}{l}\frac{d \mathbf{U}}{d t_{D}}=\mathbf{A} \mathbf{U}+\mathbf{F} \\ \left.\mathbf{U}\right|_{t_{D}=0}=\mathbf{0}\end{array}\right.$

where,

$$
\mathbf{U}=\left(\begin{array}{c}
\tilde{P}_{f} \\
\tilde{P}_{m}
\end{array}\right)
$$$$
\mathbf{A}=\left(\begin{array}{cc}
-\frac{\gamma+\lambda}{\omega} & \frac{\lambda}{\omega} \\
\frac{\lambda}{1-\omega} & -\frac{\lambda}{1-\omega}
\end{array}\right),
$$

$\mathbf{F}=\left(\begin{array}{l}\frac{T\left[h\left(x_{D}, y_{D}, z_{D}\right)\right]}{V_{\text {source }, D}} \\ 0\end{array}\right)$

\subsection{Solutions of the Models}

The solution of initial value problem (14) can be expressed as,

$$
\begin{aligned}
& \mathbf{U}=\frac{(1-\omega) \omega T\left[h\left(x_{D}, y_{D}, z_{D}\right)\right]}{\lambda \sqrt{W} V_{\text {source, },}} \\
& \int_{0}^{t_{D}}\left(\begin{array}{l}
\frac{\lambda}{\omega}\left[\left(\frac{\gamma+\lambda}{\omega}+J_{2}\right) e^{J_{1}\left(t_{D}-\tau_{D}\right)}-\left(\frac{\gamma+\lambda}{\omega}+J_{1}\right) e^{J_{2}\left(t_{D}-\tau_{D}\right)}\right] \\
\left(\frac{\gamma+\lambda}{\omega}+J_{1}\right)\left(\frac{\gamma+\lambda}{\omega}+J_{2}\right)\left(e^{J_{1}\left(t_{D}-\tau_{D}\right)}-e^{J_{2}\left(t_{D}-\tau_{D}\right)}\right)
\end{array}\right) d \tau_{D}
\end{aligned}
$$

where $W=[(1-\omega) \gamma+\lambda]^{2}-4 \gamma \lambda \omega(1-\omega)$,

$J_{1,2}=\frac{-[(1-\omega) \gamma+\lambda] \pm \sqrt{W}}{2 \omega(1-\omega)}\left(J_{2}>J_{1}\right)$

Calculate the integral of Eq. (15)

$\tilde{P}_{f}=\frac{T\left[h\left(x_{D}, y_{D}, z_{D}\right)\right]}{V_{\text {source }, D}} R\left(k, m, n, t_{D}\right)$

where $R\left(\gamma, t_{D}\right)$ is a time dependent function that related to storativity and flow capacity ratio,

$R\left(\gamma, t_{D}\right)= \begin{cases}t_{D}-\frac{(1-\omega)^{2}}{\lambda}\left(e^{J_{t_{D}}}-1\right) & \gamma=0 \\ \frac{(1-\omega)}{\sqrt{W}}\left[\left(\frac{\gamma+\lambda}{\omega J_{1}}+\frac{J_{2}}{J_{1}}\right)\left(e^{J_{t_{D}}}-1\right)-\left(\frac{\gamma+\lambda}{\omega J_{2}}+\frac{J_{1}}{J_{2}}\right)\left(e^{J_{2} t_{D}}-1\right)\right] & \gamma \neq 0\end{cases}$

Apply inverse transformation formula (13), the dimensionless pressure of natural fractures at an arbitrary point $\mathbf{r}_{D}$ $\left(x_{D}, y_{D}, z_{D}\right)$ in the reservoir is,

$P_{f D}\left(\mathbf{r}_{D}, t_{D}\right)=q_{D} A_{\text {source }}\left(\mathbf{r}_{D}, t_{D}\right)$

$$
A_{\text {source }}\left(\mathbf{r}_{D}, t_{D}\right)=\frac{1}{V_{\text {source }, D}} \sum_{k, m, n=0}^{\infty} R\left(\gamma, t_{D}\right) G_{\text {source, }, k}\left(x_{D}\right) G_{\text {source, },}\left(y_{D}\right) G_{\text {source, },}\left(z_{D}\right)
$$

where $A_{\text {source }}\left(\mathbf{r}_{D}, t_{D}\right)$ presents the dimensionless pressure at $\mathbf{r}_{D}$ caused by unit strength source; $G_{\text {source, } k}\left(l_{D}\right)$ is a function defined by properties of the source and boundary of reservoir

$G_{\text {source } e, k}\left(l_{D}\right)= \begin{cases}\frac{2 w_{I D}}{l_{e D}} & k=0 \\ \frac{2 \cos \frac{k \pi}{l_{e D}} l_{D}\left[\sin \frac{k \pi}{l_{e D}}\left(c_{I D}+w_{I D}\right)-\sin \frac{k \pi}{l_{e D}}\left(c_{I D}-w_{I D}\right)\right]}{k \pi} & k \neq 0\end{cases}$

Compared to the traditional point source function (First solution in Table 3A) derived by Ozkan et al. [21], volumetric source function Eq. (19) has more clear expression. Furthermore, Ozkan' point source solution could be diverged, and could only be calculated by transformed formation [22]. The orthogonal transformation employed to derive the volumetric source solution can avoid the complicated numerical inversion of Laplace transformation. All the advantages could simplify the programming calculation.

\subsection{Solution for Variable Flow Rate}

For the situation that the flow rate $q$ is time dependent, the solution can be obtained by Duhamel's principle,

$$
P_{f D}\left(\mathbf{r}_{D}, t_{D}\right)=\int_{0}^{t_{D}} q_{D}\left(\tau_{D}\right)\left[-\frac{d A_{\text {source }}\left(\mathbf{r}_{D}, t_{D}-\tau_{D}\right)}{d \tau_{D}}\right] d \tau_{D}
$$

The integral and differentiate in Eq. (21) are calculated numerically. Discrete dimensionless time by the step $\Delta t_{D}$, Eq. (21) can be calculated step by step. Starting from the first time interval $\left(\left[0, t_{D, 1}\right]\right)$, Eq. (21) changes to,

$$
P_{f D}\left(\mathbf{r}_{D}, t_{D, 1}\right)=q_{D}\left(t_{D}^{1}\right) A_{\text {source }}\left(\mathbf{r}_{D}, \Delta t_{D}\right)
$$

For the $\mathrm{j}$-th time interval $\left(\left[t_{D, j-1}, t_{D, j}\right], j>2\right)$, Eq. (21) changes to

$$
P_{f D}\left(\mathbf{r}_{D}, t_{D}^{j}\right)=\sum_{i=1}^{j} q_{D}\left(t_{D}^{i}\right)\left[A_{\text {saurce }}\left(\mathbf{r}_{D},(j-i-1) \Delta t_{D}\right)-A_{\text {source }}\left(\mathbf{r}_{D},(j-i) \Delta t_{D}\right)\right]
$$

where $A_{\text {source }}\left(\mathbf{r}_{D}, 0\right)=0$.

\section{FLOW MODEL OF HYDRAULIC FRACTURE}

\subsection{Assumption of Flow in Hydraulic Fracture}

The length of hydraulic fracture is $x_{F}$, height is $y_{F}$, width is $w_{F}$. The flow in hydraulic fracture is only occurring on the plane of the fracture. Oil from reservoir flows into the well through the hydraulic fracture immediately, the pressure of hydraulic fracture recovers to balance at each time step.

Divide hydraulic fracture into $M \times N$ segments (M segments in $x$ direction and $\mathrm{N}$ segments in $y$ direction, both $\mathrm{M}$ and $\mathrm{N}$ are even). The $i$-th segment is denoted as $F_{\text {source }, i}$, where central coordinate is $\left(x_{i}, y_{i}\right)$, total rate is $q_{F, i}$, volume is $V_{i}\left(V_{i}=2 w_{F x, i} \times 2 w_{F y, i} \times w_{F}\right)$. The central coordinate of horizontal well which intersected with the hydraulic fracture is $\left(x_{w}, y_{w}\right)$, the volume is $V_{w}$ 
$\left(V_{w}=4 w^{2} \times w_{F}, w=\sqrt{\frac{\pi r_{w}^{2}}{4}}\right)$.

Fig. (2) shows an example of hydraulic fracture which is divided in to $4 \times 2$ segments.

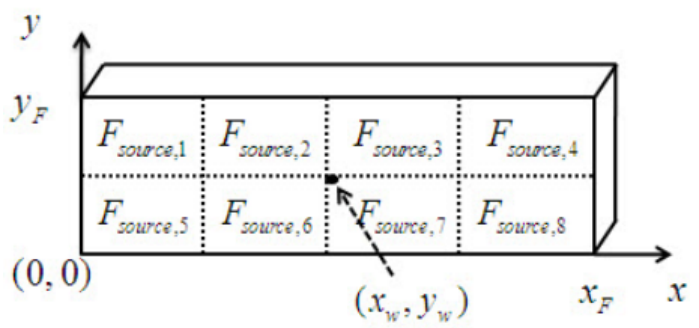

Fig. (2). Cartesian coordinate system of hydraulic fractures (divided into $4 \times 2$ segments).

\subsection{Pressure Drop in Hydraulic Fracture}

Assume only the i-th segment $F_{i}$ flows to the well, the same expressions of dimensionless variables are defined as before, flow model of hydraulic fracture is,

$\frac{\partial^{2} P_{F D}}{\partial x_{D}^{2}}+\frac{\partial^{2} P_{F D}}{\partial y_{D}^{2}}+\frac{q_{F, i D} k_{f}}{V_{\text {source }, D} k_{F}}\left[\frac{V_{\text {source }, D} h_{w}\left(x_{D}, y_{D}\right)}{V_{w}}-h_{i}\left(x_{D}, y_{D}\right)\right]=0$

$\left.P_{F D}\right|_{t=0}=0$

$\left.\frac{\partial P_{F D}}{\partial x_{D}}\right|_{x_{D}=0, x_{F D}}=0$

$\left.\frac{\partial P_{F D}}{\partial y_{D}}\right|_{y_{D}=0, y_{F D}}=0$

where

$$
\begin{aligned}
& h_{i}\left(x_{D}, y_{D}\right)=\left[H\left(x_{D}-x_{i D}-w_{F x, i D}\right)-H\left(x_{D}-x_{i D}+w_{F x, i D}\right)\right] \times \\
& {\left[H\left(y_{D}-y_{i D}-w_{F y, i D}\right)-H\left(y_{D}-y_{i D}+w_{F y, i D}\right)\right]} \\
& h_{w}\left(x_{D}, y_{D}\right)=\left[H\left(x_{D}-x_{w D}-w_{w D}\right)-H\left(x_{D}-x_{w D}+w_{w D}\right)\right] \times \\
& {\left[H\left(y_{D}-y_{w D}-w_{w D}\right)-H\left(y_{D}-y_{w D}+w_{w D}\right)\right]}
\end{aligned}
$$

Corresponding to model $(24) \sim(27)$, define the orthogonal transformation as,

$$
\tilde{P}_{F}=T_{F}\left(P_{F D}(x, y)\right)=\int_{0}^{x_{F D}} \int_{0}^{y_{F D}} P_{F D} \cos \frac{k \pi}{x_{F D}} x_{D} \cos \frac{m \pi}{y_{F D}} y_{D} d x_{D} d y_{D}
$$

Inverse transformation formula is,

$$
P_{F D}\left(x_{D}, y_{D}\right)=\sum_{k, m=1}^{\infty} \frac{F_{k, m}\left(x_{D}, y_{D}\right)}{\left\|F_{k, m}\right\|^{2}} \tilde{P}_{F}
$$

Where characteristic function of the orthogonal transformation is

$F_{k, m}\left(x_{D}, y_{D}\right)=\cos \frac{k \pi}{x_{F D}} x_{D} \cos \frac{m \pi}{y_{F D}} y_{D}$

Characteristic value is,
$\gamma_{F}=\left(\frac{k \pi}{x_{F D}}\right)^{2}+\left(\frac{m \pi}{y_{F D}}\right)^{2}$

Norm of characteristic function is,

$\left\|F_{k, m}\right\|^{2}=\frac{x_{F D} y_{F D}}{4}$

Application of the inverse transformation formula (29), dimensionless pressure in hydraulic fracture is,

$P_{F D}\left(x_{D}, y_{D}\right)=q_{F, i D} R_{i}\left(x_{D}, y_{D}\right)$

Where

$$
\begin{aligned}
& T_{k, i}=\frac{x_{F D}}{k \pi}\left[\sin \frac{k \pi}{x_{F D}}\left(x_{i D}+w_{F x, i D}\right)-\sin \frac{k \pi}{x_{F D}}\left(x_{i D}-w_{F x, i D}\right)\right] \\
& T_{m, i}=\frac{y_{F D}}{m \pi}\left[\sin \frac{m \pi}{y_{F D}}\left(y_{i D}+w_{F y, i D}\right)-\sin \frac{m \pi}{y_{F D}}\left(y_{i D}-w_{F y, i D}\right)\right] \\
& T_{k, w}=\frac{x_{F D}}{k \pi}\left[\sin \frac{k \pi}{x_{F D}}\left(x_{w D}+w_{w D}\right)-\sin \frac{k \pi}{x_{F D}}\left(x_{w D}-w_{w D}\right)\right] \\
& T_{m, i}=\frac{y_{F D}}{m \pi}\left[\sin \frac{m \pi}{y_{F D}}\left(y_{w D}+w_{w D}\right)-\sin \frac{m \pi}{y_{F D}}\left(y_{w D}-w_{w D}\right)\right] \\
& R_{i}\left(x_{D}, y_{D}\right)=\frac{k_{f}}{V_{\text {source }, D} k_{F}} \sum_{k, m=1}^{\infty} \frac{\left[F_{k, m}\left(x_{w D}, y_{w D}\right)-F_{k, m}\left(x_{D}, y_{D}\right)\right]}{\gamma_{F}\left\|F_{k, m}\right\|^{2}}\left(\frac{T_{k, w} T_{m, w} V_{\text {saurce }, D}}{V_{w D}}-T_{k, i} T_{m, i}\right)
\end{aligned}
$$

According to the superposition theory, when all the segments of hydraulic fracture flow to the well, dimensionless pressure of hydraulic fractures is,

$$
P_{F D}\left(x_{D}, y_{D}\right)=\sum_{i=1}^{N \times M} q_{F, i D} R_{i}\left(x_{D}, y_{D}\right)
$$

\section{PRODUCTIVITY OF FRACTURED HORIZONTAL WELL}

In this section, productivities of fractured horizontal wells for cased-hole and open-hole are discussed. The main difference is fluids flow to the horizontal well is considered for open-hole case, but not for cased-hole case.

\subsection{Productivity of Fractured Cased-hole Horizontal Well}

For fractured cased-hole horizontal well, fluids only flow to hydraulic fractures. Assume that there are $N_{H}$ hydraulic fractures in the reservoir, the $\mathrm{j}$-th hydraulic fracture is divided into $(N \times M)^{(j)}$ segments, the size is $\left(w_{F}^{(j)}, x_{F}^{(j)}, y_{F}^{(j)}\right)$. Then the reservoir exists $N_{t}$,

$\left(N_{t}=\sum_{j=1}^{N_{H}}(N \times M)^{(j)}\right)$

volumetric source, 3-D coordinate of central coordinate is $\left(\mathbf{r}_{1 D}, \mathbf{r}_{2 D}, \cdots, \mathbf{r}_{N_{t} D}\right), \quad 2-\mathrm{D}$ coordinate is $\left(\mathbf{r}_{1 D}^{F}, \mathbf{r}_{2 D}^{F}, \cdots, \mathbf{r}_{N_{t} D}^{F}\right)$. Fig. (3) shows the schematic and flow direction of casedhole case. 


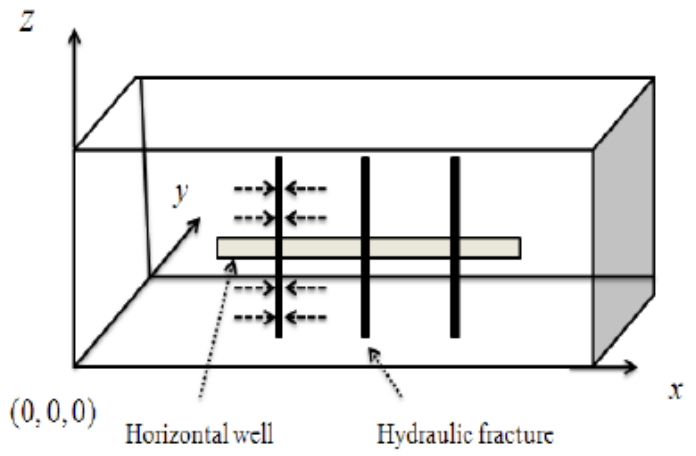

Fig. (3). Schematic of fractured cased-hole horizontal well (3 hydraulic fractures).

According to the superposition theory, for the $N_{t}$ volumetric source situation, dimensionless pressure is,

$P_{f D}\left(\mathbf{r}_{D}, t_{D}^{1}\right)=\sum_{s=1}^{N_{t}} q_{s D}\left(t_{D}^{1}\right) A_{s}\left(\mathbf{r}_{D}, \Delta t_{D}\right)$

$P_{f D}\left(\mathbf{r}_{D}, t_{D}^{j}\right)=\sum_{s=1}^{N_{t}} \sum_{i=1}^{j} q_{s D}\left(t_{D}^{j-1}\right)\left[A_{s}\left(\mathbf{r}_{D},(j-i) \Delta t_{D}\right)-A_{s}\left(\mathbf{r}_{D},(j-i-1) \Delta t_{D}\right)\right]$

$(j \geq 2)$

For the j-th time interval, matrix of each source's rate is $\mathbf{q}_{D}^{j}=\left(q_{1 D}^{j}, q_{2 D}^{j}, \cdots, q_{N_{t} D}^{j}\right)$, which can be calculated by the following $N_{t}$-order equation system,

$$
\left(\mathbf{A}_{1}+\mathbf{A}_{2}\right) \mathbf{q}_{j}=\mathbf{b}_{j}
$$

Where,

$\mathbf{b}_{\mathbf{j}}=\left(P_{w D}, P_{w D}, \cdots, P_{w D}\right)^{T}-\Delta \mathbf{P}_{\mathbf{j}}, \mathbf{A}_{\mathbf{1}}=\left(a_{m n}^{(1)}\right)_{N_{t} \times N_{t}}$,

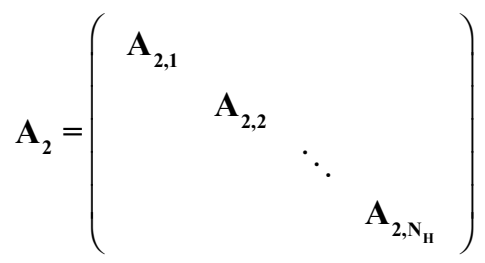

for $m, n=1,2, \cdots, N_{t}$, each elements in matrix $\mathbf{A}_{1}, \mathbf{A}_{2}$ and vector $\Delta \mathbf{P}_{\mathrm{j}}$ is,

$$
\begin{aligned}
& \mathbf{A}_{2, \mathbf{k}}=\left(a_{m n}^{2, k}\right)_{(N \times M)^{(k)} \times(N \times M)^{(k)}} \\
& \Delta \mathbf{P}_{\mathbf{j}}(m)= \begin{cases}0 & j=1 \\
\sum_{s=1}^{N_{h}} q_{s D}\left(t_{D}^{1}\right)\left[A_{s}\left(\mathbf{r}_{m D}, 2 \Delta t_{D}\right)-A_{s}\left(\mathbf{r}_{m D}, \Delta t_{D}\right)\right] & j=2 \\
\sum_{s=1}^{N_{h}} \sum_{i=1}^{j=1} q_{s D}\left(t_{D}^{j}\right)\left[A_{s}\left(\mathbf{r}_{m D},(j-i+1) \Delta t_{D}\right)-A_{s}\left(\mathbf{r}_{m D},(j-i) \Delta t_{D}\right)\right] & j>2\end{cases} \\
& a_{m n}^{(1)}=A_{n}\left(\mathbf{r}_{m D}, \Delta t_{D}\right) \\
& \mathbf{A}_{2, \mathbf{k}}=\left(a_{m n}^{2, k}\right)_{(N \times M)^{(k)} \times(N \times M)^{(k)}}, a_{m, n}^{2, k}=R_{J}\left(\mathbf{r}_{F I}\right)
\end{aligned}
$$

$$
\begin{aligned}
& I=\left\{\begin{array}{ll}
i & k=1 \\
\sum_{l=1}^{k-1}(N \times M)^{(l)}+i & k>1
\end{array},\right. \\
& J= \begin{cases}j & k=1 \\
\sum_{l=1}^{k-1}(N \times M)^{(l)}+j & k>1\end{cases}
\end{aligned}
$$

The matrix of each source's rate $\mathbf{q}_{D}^{j}$ can be calculated, the total flow rate of the well $Q_{D}^{j}$ is,

$$
Q_{D}^{j}=\sum_{m=1}^{N_{t}} q_{m D}^{j}
$$

\subsection{Productivity of Fractured Open-hole Horizontal Well}

For fractured open-hole horizontal well, productivity of both horizontal well and hydraulic fractures should be considered. Dividing the horizontal well into $N_{H w}$ segments, 3D coordinate of central coordinates of additional volumetric sources are $\left(\mathbf{r}_{N,+1, D}, \mathbf{r}_{N+2, D}, \cdots, \mathbf{r}_{N_{1}+N_{H Y}, D}\right)$. Fig. (4) shows the schematic and flow direction of open-hole case.

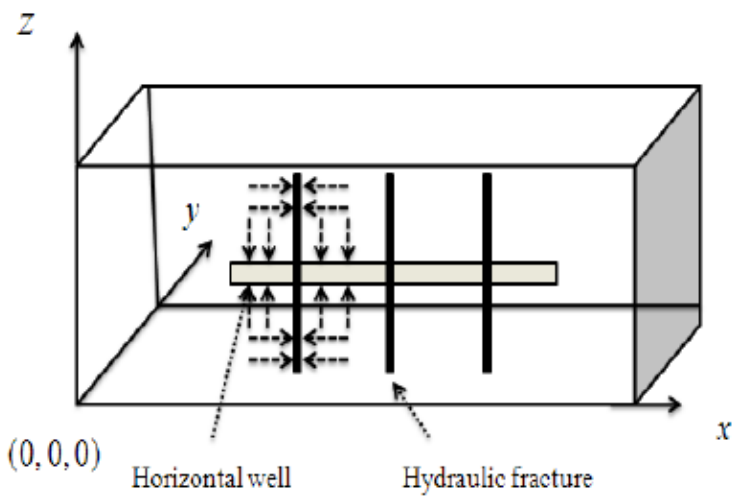

Fig. (4). Schematic of fractured open-hole horizontal well (3 hydraulic fractures).

After applying superposition theory, dimensionless pressure of natural fractures is,

$$
\begin{aligned}
& P_{f D}\left(\mathbf{r}_{D}, t_{D}^{1}\right)=\sum_{s=1}^{N_{t}+N_{H W}} q_{s D}\left(t_{D}^{1}\right) A_{s}\left(\mathbf{r}_{D}, \Delta t_{D}\right) \\
& P_{f D}\left(\mathbf{r}_{D}, t_{D}^{j}\right)=\sum_{s=1}^{N_{t}+N_{H W}} \sum_{i=1}^{j} q_{s D}\left(t_{D}^{j-1}\right)\left[A_{s}\left(\mathbf{r}_{D},(j-i) \Delta t_{D}\right)-A_{s}\left(\mathbf{r}_{D},(j-i-1) \Delta t_{D}\right)\right]
\end{aligned}
$$

$(j \geq 2)$

For the $\mathrm{j}$-th time interval, matrix of each source's rate is $\mathbf{q}_{D}^{j}=\left(q_{1 D}^{j}, q_{2 D}^{j}, \cdots, q_{N_{t} D}^{j}, q_{\left(N_{t}+1\right) D}^{j}, \cdots, q_{\left(N_{t}+N_{H W}\right) D}^{j}\right)$, which can be calculated by the following $N_{t}+N_{H W}$-order equation system

$$
\left(\overline{\mathbf{A}}_{1}+\overline{\mathbf{A}}_{2}\right) \mathbf{q}_{j}=\overline{\mathbf{b}}_{j}
$$

where, 
$\overline{\mathbf{b}}_{\mathbf{j}}=\left(P_{w D}, P_{w D}, \cdots, P_{w D}\right)^{T}-\Delta \overline{\mathbf{P}}_{\mathbf{j}}, \overline{\mathbf{A}}_{1}=\left(a_{m n}^{(1)}\right)_{\left(N_{t}+N_{H W}\right) \times\left(N_{t}+N_{H W}\right)}$,

$\overline{\mathbf{A}}_{2}=\left(\begin{array}{cc}\mathbf{A}_{2} & \mathbf{0} \\ \mathbf{0} & \mathbf{0}\end{array}\right)$

for $m, n=1,2, \cdots, N_{t}+N_{H W}$, each elements in matrix $\mathbf{A}_{1}$ and vector $\Delta \overline{\mathbf{P}}_{\mathbf{j}}$ is,

$\Delta \overline{\mathbf{P}}_{\mathbf{j}}(m)= \begin{cases}0 & j=1 \\ \sum_{s=1}^{N_{t}+N_{H W}} q_{s D}\left(t_{D}^{1}\right)\left[A_{s}\left(\mathbf{r}_{m D}, 2 \Delta t_{D}\right)-A_{s}\left(\mathbf{r}_{m D}, \Delta t_{D}\right)\right] & j=2 \\ \sum_{s=1}^{N_{t}+N_{H W}} \sum_{i=1}^{j=1} q_{s D}\left(t_{D}^{j}\right)\left[A_{s}\left(\mathbf{r}_{m D},(j-i+1) \Delta t_{D}\right)-A_{s}\left(\mathbf{r}_{m D},(j-i) \Delta t_{D}\right)\right] & j>2\end{cases}$

$a_{m n}^{(1)}=A_{n}\left(\mathbf{r}_{m D}, \Delta t_{D}\right)$

The matrix of each source's rate $\mathbf{q}_{D}^{j}$ can be calculated, the total flow rate of the well $Q_{D}^{j}$ is,

$Q_{D}^{j}=\sum_{s=1}^{N_{t}+N_{H W}} q_{s D}^{j}$

The methods to calculate productivity of hydraulic fractured open-hole and cased-hole horizontal wells are derived by volumetric source functions. It is the basic theory in predicting production optimal designing of hydraulic fracturing.

\subsection{Comparison between Two Cases}

An example is simulated to should the difference between two cases. Horizontal is placed at the central of reservoir, and hydraulic fractures are assumed to be equally spaced along the horizontal well. Table 1 shows the basic parameter values of this simulation.

Before analyzing difference of two cases, we compare the results of volumetric source model with the analytical flow model [1] to show the validity (see Fig. 5). The casedhole case is employed to accomplish the comparison; and in the calculation of analytical flow model, length horizontal well equals to length of reservoir $\left(x_{e}=L_{H}=500 \mathrm{~m}\right)$.

As shown in Fig. (6), difference of two cases is obvious only at the beginning. Earlier the flow rate of open-hole case is much greater than other the interference between the sources is not serious to affect productivity of each source, the open-hole case takes advantage the sources of horizontal well.

Fig. (7) indicates ratio of two cases flow rate. Before the 10-th day, the ratio is less than 1 , since flow rate of the openhole case is greater than another. In this time interval, horizontal well has contribution on productivity, it shows the advantage caused by horizontal well. As the contribution of horizontal well cannot offset the loss of hydraulic fracture, the curve keep raising to a highest point (at the $50^{\text {th }}$ day), which is the disadvantage caused by horizontal well. Time node (55-th day) of the highest point illustrates the duration that horizontal well affects the productivity. Then the curve keeps decreasing to 1 , for the existence horizontal well has less effect on production and causes additional pressure drop. The curve is employed to show the difference of two cases, when the curve is smoother, the difference is smaller.

\section{INFLUENCES OF HYDRAULIC FRACTURES AND RESERVOIR}

The comparison of two cases shows mechanisms that cause the difference of productivity: horizontal well's production and additional pressure drop. Flow rates of openhole case and cased hole-case are different, degrees of hydraulic fractures and reservoir' factors affect the productivity are analyzed in the section.

\subsection{Factors of Hydraulic Fracture}

Figs. (8-10) show the effect of hydraulic fracture's factors on cumulative productions of two cases for 100 days. As

Table 1. Basic data used for simulation.

\begin{tabular}{|c|c|c|c|}
\hline Parameter & Data & Parameter & 5 \\
\hline \hline$x_{e}(\mathrm{~m})$ & 1000 & $N_{H}$ & 400 \\
\hline$y_{e}(\mathrm{~m})$ & 500 & $x_{F}(\mathrm{~m})$ & 10 \\
\hline$z_{e}(\mathrm{~m})$ & 20 & $y_{F}(\mathrm{~m})$ & 0.003 \\
\hline$L_{H}(\mathrm{~m})$ & 500 & $w_{F}(\mathrm{~m})$ & 50 \\
\hline$r_{w}(\mathrm{~m})$ & 0.05 & $k_{F}\left(\mu \mathrm{m}^{2}\right)$ & 20 \\
\hline$k_{m}\left(\mu \mathrm{m}^{2}\right)$ & 0.005 & $p_{i}(\mathrm{MPa})$ & 10 \\
\hline$\phi_{m}$ & 0.15 & $p_{w}(\mathrm{MPa})$ & 2 \\
\hline$k_{f}\left(\mu \mathrm{m}^{2}\right)$ & 1 & $\mu(\mathrm{mPa} \cdot \mathrm{s})$ & 1.08 \\
\hline$V_{f}$ & 0.001 & $c_{t}(1 / \mathrm{MPa})$ & 100 \\
\hline$\lambda$ & 1000 & $B$ & 0.0005 \\
\hline$\omega$ & 0.01 & $t(\mathrm{day})$ & \\
\hline
\end{tabular}




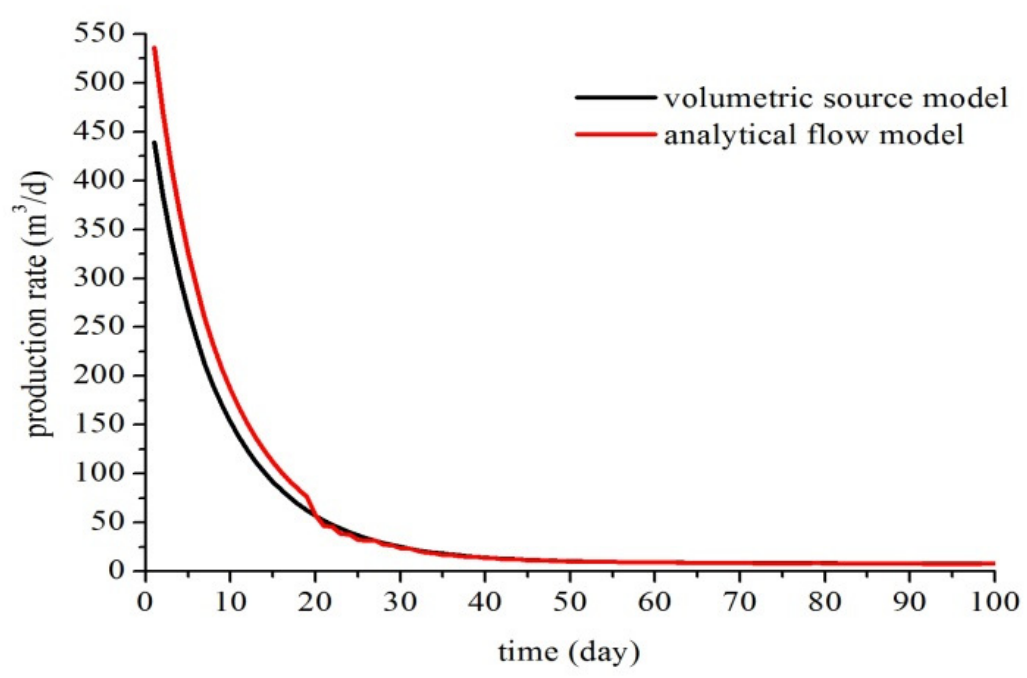

Fig. (5). Comparison of volumetric source model and analytical flow model.

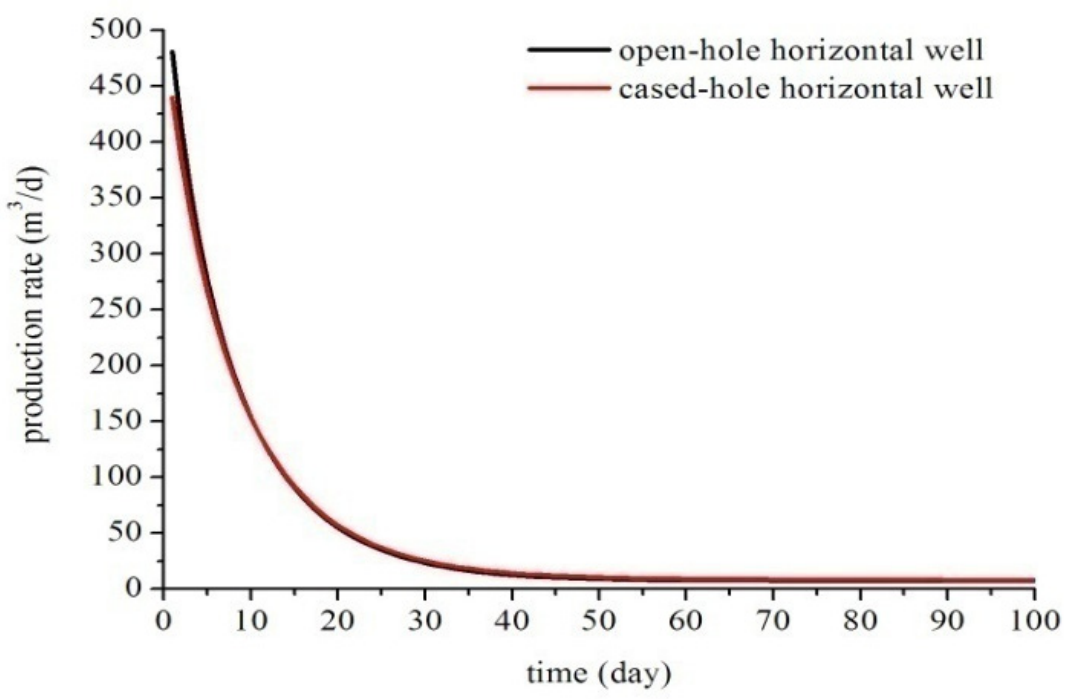

Fig. (6). Comparison between two cases.

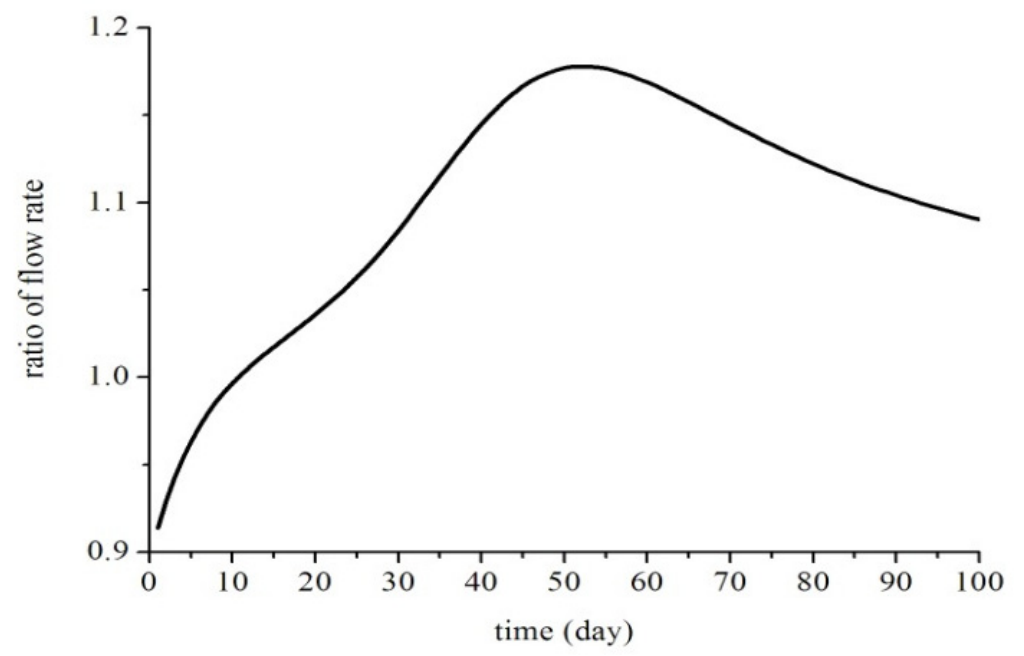

Fig. (7). Ratio of flow rate. 


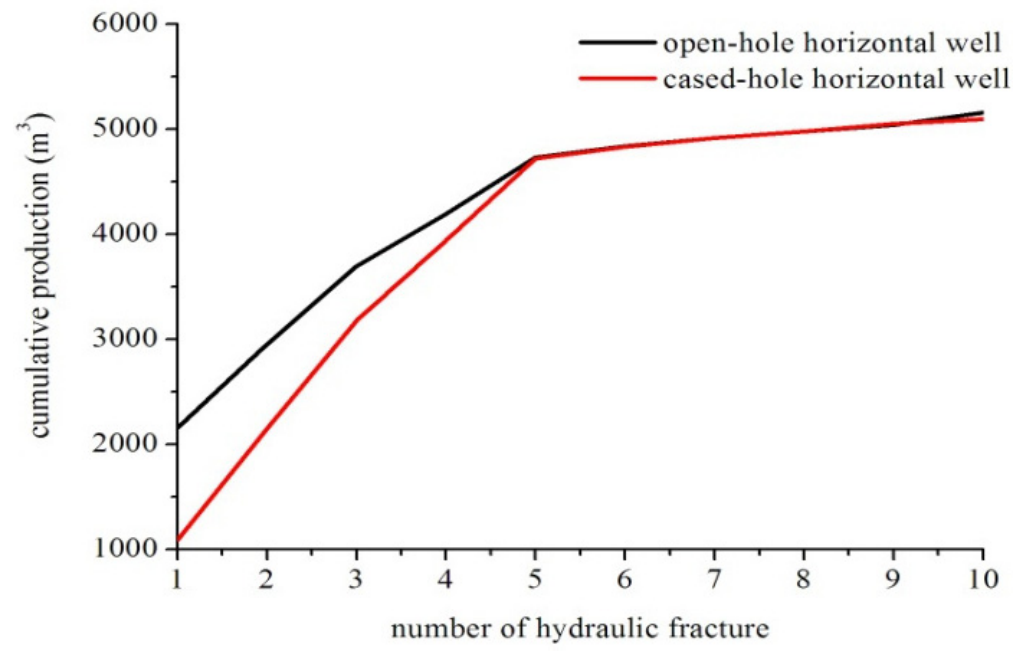

Fig. (8). Effect of the number on cumulative production.

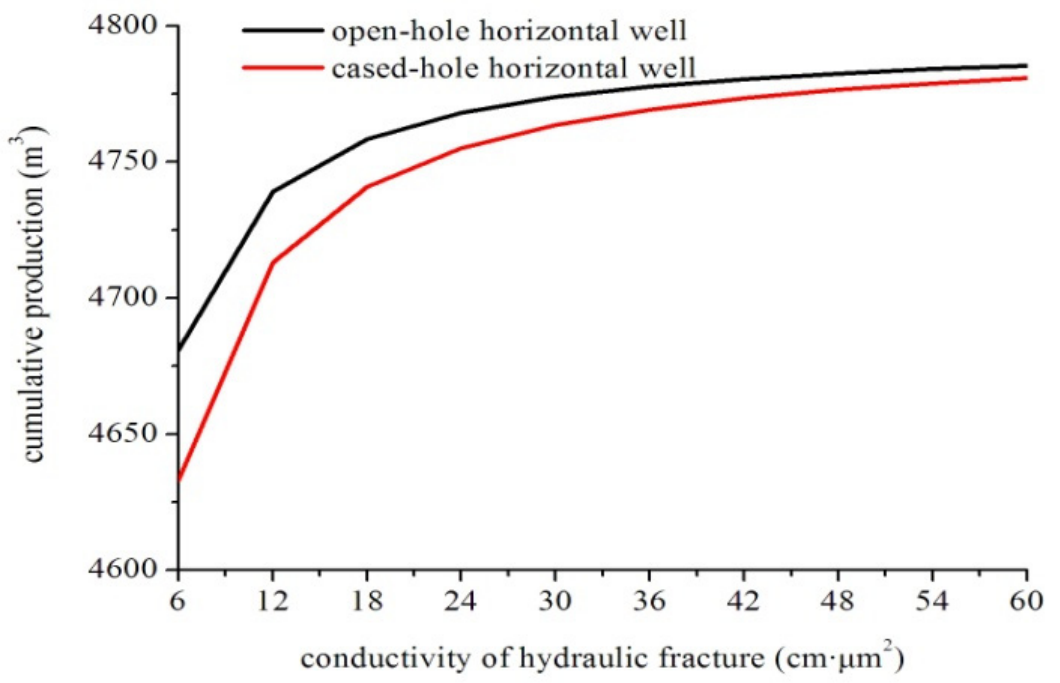

Fig. (9). Effect of the conductivity on cumulative production.

illustrated in Fig. (8), when the number is equal or greater than 5 , the cumulative productions of two cases do not increase obviously, and difference of two cases is ignorable. When the number is less than 5, the open-hole case has greater cumulative production than another. Fig. (9) shows that the cumulative production of open-hole case is higher the other and increasing conductivity can bring greater cumulative production, but the effects do not obviously as the number do. Increasing the number and conductivity, the curve of open-hole case is above than other, it means the advantage caused by horizontal well has outweighed the disadvantage. Fig. (10) shows that increasing the length of hydraulic fracture improves the cumulative production significantly for both cases, and difference of two cases is ignorable, it indicates that advantage has offset the disadvantage.

Figs. (11-13) show the effect of hydraulic fracture's factors on the ratio of flow rate. Increasing the number or conductivity of hydraulic fracture, the curve becomes smoother and the time node of the highest point shows up earlier. While as the length of hydraulic fracture increasing, the curve becomes smoother, but the time node shows up later.
Because the distances between sources of hydraulic fracture and horizontal well are farther, the elimination effect of horizontal well' source costs more time.

It is an obvious conclusion that different effect completion methods become inconspicuous when hydraulic fractures control the production, which can be achieved by increasing number, and length of conductivity of hydraulic fractures.

\subsection{Factors of Reservoir}

Fig. (14) shows the ratio of flow rate changes with different permeability of reservoir. For lower permeability of reservoir, the curve becomes smoother and the highest point shows up earlier. When the permeability is $0.0001 \mu \mathrm{m}^{2}$, the ratio approach to 1 , it indicates the flow to horizontal well could be ignored for low-permeability reservoir.

Figs. $(15,16)$ show the effects of characteristic parameter on the ratio of flow rate. For larger flow capacity ratio $\lambda$, the time node of highest point shows up earlier, the curve becomes more abrupt. Where the flow capacity ratio $\lambda$ is small 


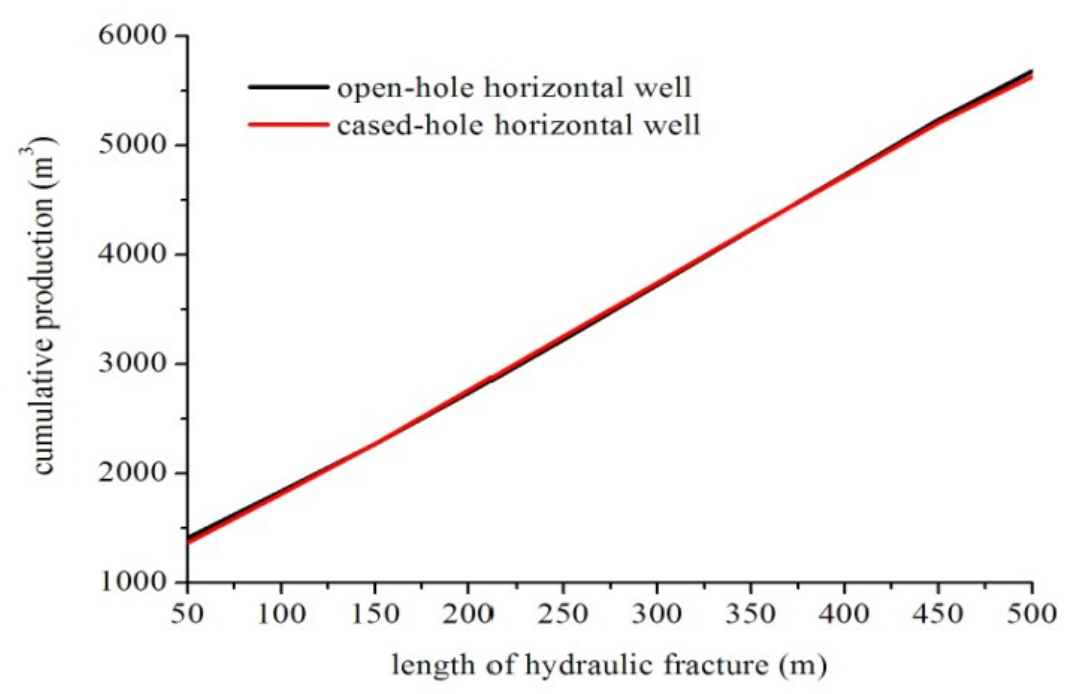

Fig. (10). Effect of the length on cumulative production.

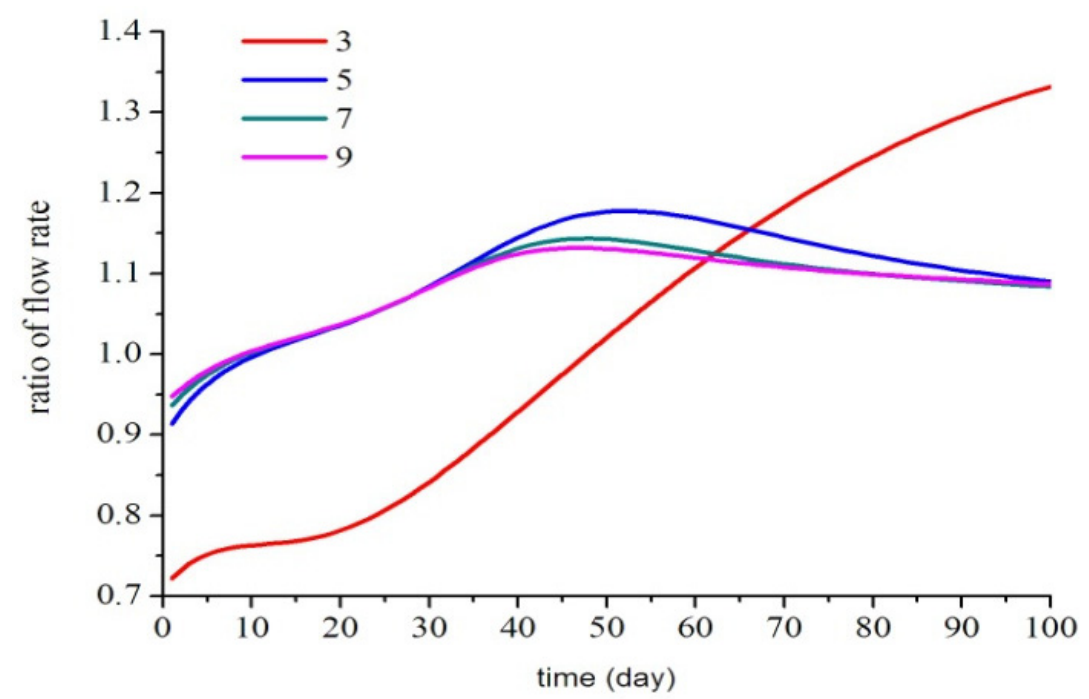

Fig. (11). Effect of the number on ratio of flow rate.

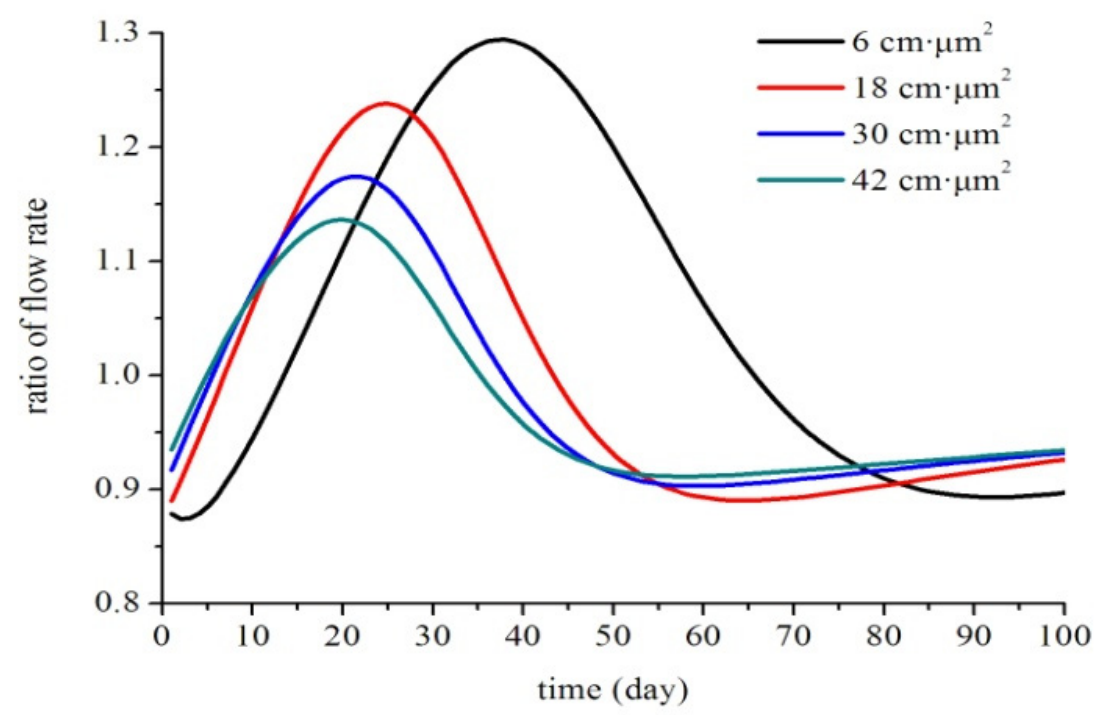

Fig. (12). Effect of the conductivity on ratio of flow rate. 


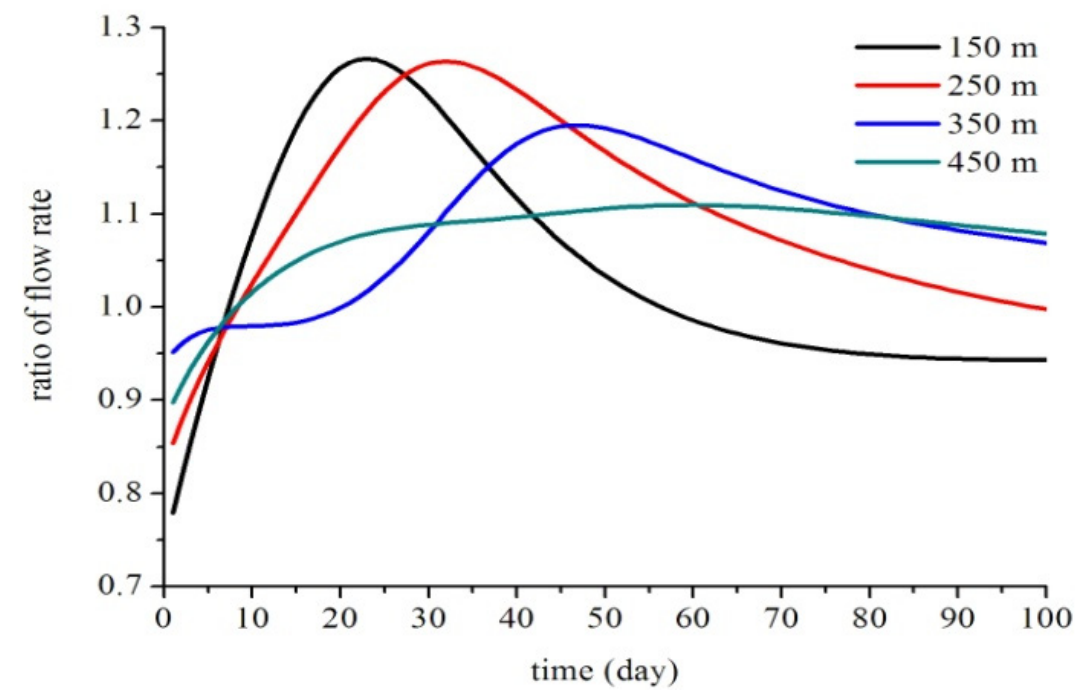

Fig. (13). Effect of the length on ratio of flow rate.

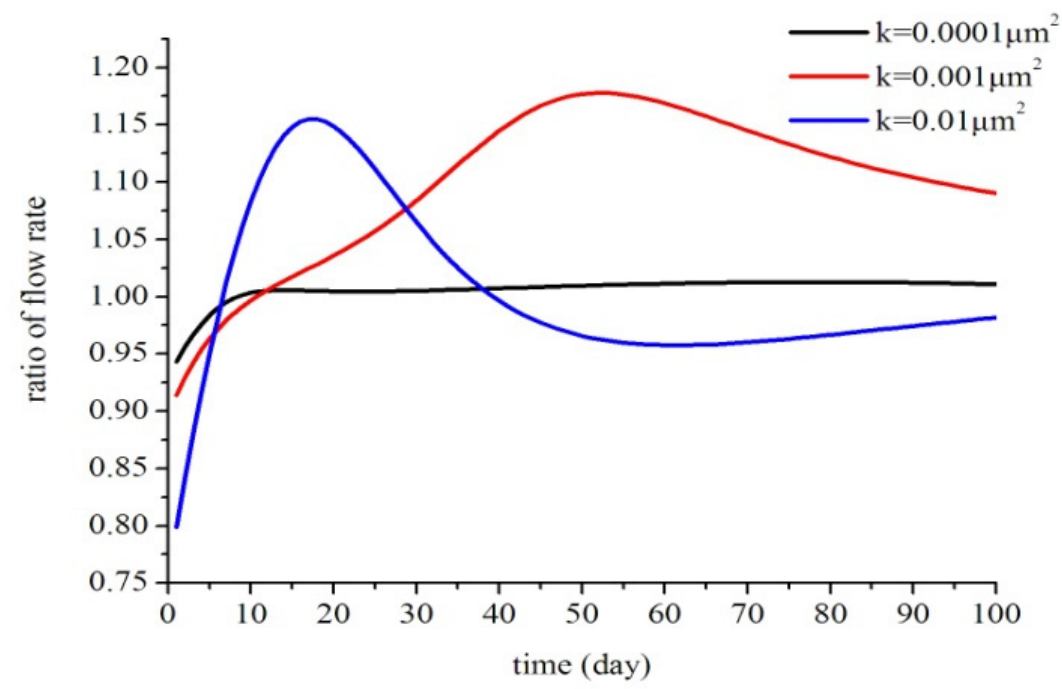

Fig. (14). Effect of the permeability of reservoir on ratio of flow rate.

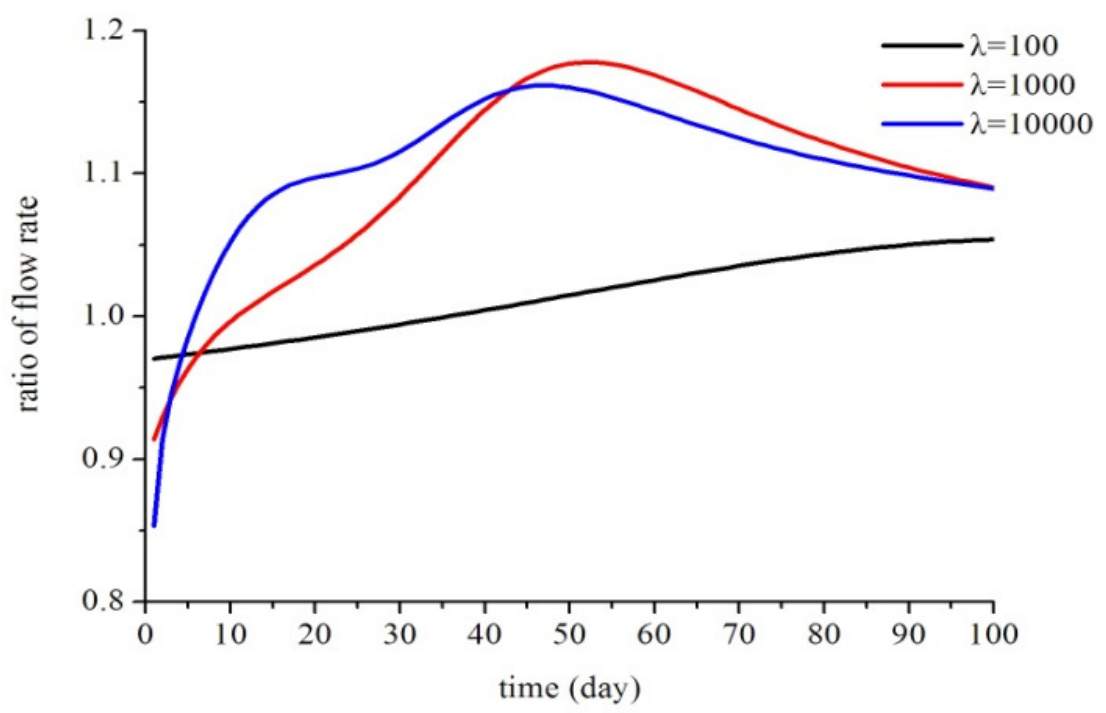

Fig. (15). Effect of the flow capacity ratio on ratio of flow rate. 


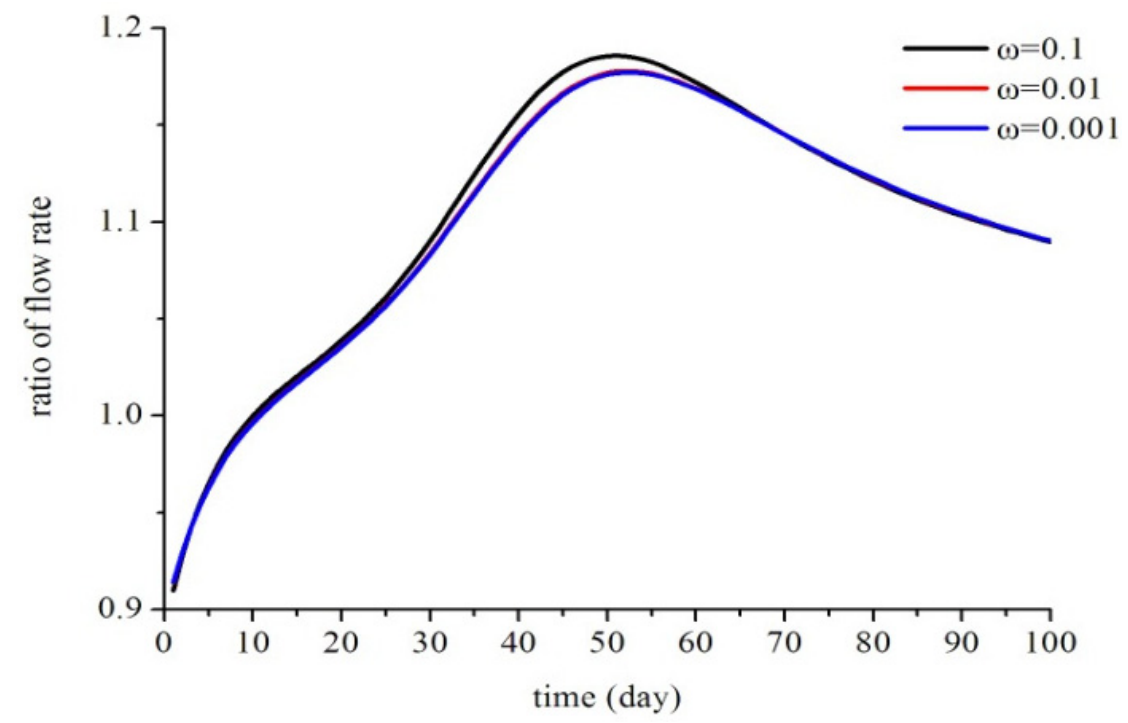

Fig. (16). Effect of the storativity on ratio of flow rate.

enough, the difference of open-hole case and cased-hole case could be eliminated. Storativity $\omega$ does not have obvious effect on the curves, the curve is a little higher for larger $\omega$.

The difference between the two cases is affected by flow capacity of reservoir. When the flow capacity of reservoir is bigger, production of horizontal well is more significant, and the difference is more obvious.

\section{CONCLUSION}

1. New volumetric source models of flow in reservoir and hydraulic fractures are developed and solved by taking orthogonal transformation. By combining two models, the productivity of hydraulic fractured open-hole and cased-hole horizontal wells can be calculated.

2. Compared to traditional point source functions, the volumetric source function has many advantages in many aspects: better property of source function, more adjustable, more convenient in programming calculation.

3. A practical example is simulated to show the difference and mechanisms of open-hole case and cased-hole case. Result show that different completion methods have huge effect on productivity for the production of horizontal wells and the additional pressure drop.

4. The difference of open-hole case and cased-hole case becomes ignorable when the number, conductivity, and length of hydraulic fractures are large enough, or permeability of reservoir and flow capacity ratio is small enough.

\section{CONFLICT OF INTEREST}

The authors confirm that this article content has no conflict of interest.

\section{ACKNOWLEDGEMENTS}

This study is supported by the National Science and Technology Major Project of China (Grant No.I, 2011 ZX05022-006-004HZ).

\section{REFERENCES}

[1] M. Brown, E. Ozkan, R.S. Raghavan, and H. Kazemi, "Practical solutions for pressure transient responses of fractured horizontal wells in unconventional reservoirs", In: SPE Annual Technical Conference and Exhibition, New Orleans, Louisiana, SPE 125043, 2009, pp. 1-18.

[2] M. Brown, E. Ozkan, R.S. Raghavan, and H. Kazemi, "Comparison of fractured-horizontal-well performance in tight sand and shale reservoirs", In: SPE Reservoir Evaluation \& Engineering, Golden, CO, United States, 2012, pp. 248-59.

[3] A. Hasan, "A Triple-porosity Model for Fractured Horizontal Wells", M.SC Thesis, A \& M University, Texas, 2010.

[4] S.K. Siddiqui, and A.H. Dehghanpour, "New advances in production data analysis of hydraulically fractured tight reservoirs", In: SPE Canadian Unconventional Resources Conference, SPE 162830, Alberta, Canada, 2012, pp. 1-38.

[5] R. Raghavan, and C.C. Chih, "An analysis of horizontal wells intercepted by multiple fractures", SPE Journal, vol. 2, pp. 235-45, 1994.

[6] C.C. Chih, and R. Raghavan, "A multiply-fractured horizontal well in a rectangular drainage region”, SPE Journal, vol. 2, pp. 455-65, 1997.

[7] A. Zerzar, and Y. Bettam, "Interpretation of multiple hydraulically fractured horizontal well in closed systems", In: Canadian International Petroleum Conference, Kuala Lumpur, Malaysia, 2004, pp. $1-14$.

[8] J. Guo, F. Zeng, and J. Zhao, "A new model to predict fractured horizontal well production”, In: Canadian International Petroleum Conference, Calgary, Alberta, 2006, pp. 1-8.

[9] F.H. Zeng, J.C. Guo, and J. Yi, "An unsteady state computation model of fractured horizontal well coupling with reservoir", Geoscience, vol. 25, pp. 1159-65, 2011.

[10] P.Q. Lian, L.S. Cheng, and J.Y. Cui, "A new computation model of fractured horizontal well coupling with reservoir", International Journal for Numerical Methods in Fluids, vol. 67, pp. 1047-56, 2012.

[11] J.J. Lin, and D. Zhu, "Modeling well performance for fractured horizontal gas well", In: CPS/SPE International Oil \& Gas Conference and Exhibition, SPE 130794, 2010.

[12] J.J. Lin, and D. Zhu, "Predicting well performance in complex fracture systems by slab source method", In: SPE Hydraulic Fracturing Technology Conference, Texas, USA, SPE 151960, 2012.

[13] H. Yunsuk, J.J. Lin, D. Zhu, and S. David, "Predicting fractured well performance in naturally fractured reservoir", In: International Petroleum Technology Conference, A\&M University, Texas, SPE 17010, 2013.

[14] A.C. Gringarten, J.R. Ramey, and J. Henry, "The use of source and Green's functions in solving unsteady-flow problems in reservoir", SPE Journal, vol. 13, pp. 285-296, 1973. 
[15] B.O. Ogunsanya, T.P. Oetama, and J.F. Lea, "A coupled model for analyzing transient pressure behavior of horizontal drain-holes", In: SPE Production and Operations Symposium, SPE 94331, 2005, pp. $1-14$.

[16] B.O. Ogunsanya, T.P. Oetama, and J.F. Lea, "A robust type curve solution for analyzing pressure-transient behaviors of both vertical and horizontal fractures systems", In: Annual SPE International Technical Conference and Exhibition, Abuja, Nigeria, SPE 105979, 2006, pp. 1-15.

[17] P.P. Valko, and S. Amini, "The method of distributed volumetric sources for calculating the transient and pseudosteady-state productivity of complex well-fracture configurations", In: SPE Hydraulic Fracturing Technology Conference, Texas, U.S.A, SPE 106279, 2007, pp. 1-14.

[18] D. Zhu, F. Magalhaes, and P.P. Valko, "Predicting productivity of multiple-fractured horizontal gas wells", In: SPE Hydraulic Frac- turing Technology Conference, Texas, U.S.A, SPE 106280, 2007, pp. 1-8.

[19] F. Magalhaes, D. Zhu, S. Amini, and P.P. Valko, "Optimization of fractured-well performance of horizontal gas wells", In: International Oil Conference and Exhibition, Veracruz, Mexico, SPE 108779, 2007, pp. 1-7.

[20] S. Amini, and P.P. Valko, "Using distributed volumetric sources to predict production from multiple-fractured horizontal wells under non-Darcy-flow conditions", SPE Journal, vol. 15, pp. 105-15, 2010 .

[21] E. Ozkan, and R.S. Raghavan, "New solutions for Well-TestAnalysis Problems: Part 1-Analytical Considerations", SPE Formation Evaluation, vol. 6, pp. 359-68, 1991.

[22] E. Ozkan, and R.S. Raghavan, "New solutions for Well-TestAnalysis Problems: Part 2-Computational Considerations and Applications", SPE Formation Evaluation, vol. 6, pp. 369-78. 1991.

Received: August 11, 2014

Revised: June 05, 2015

Accepted: July 05, 2015

(C) Wang et al.; Licensee Bentham Open.

This is an open access article licensed under the terms of the Creative Commons Attribution Non-Commercial License (http://creativecommons.org/licenses/by-nc/3.0/) which permits unrestricted, non-commercial use, distribution and reproduction in any medium, provided the work is properly cited. 